\title{
Pulsar distance measurements using interstellar scintillation data
}

\author{
T.V. Smirnova, V.I. Shishov
}

Radio Astronomy Observatory of Astro Space Center, Lebedev Physical Institute, Pushchino, Moscow region 142292, Russia

\begin{abstract}
We evaluate the distance to pulsars and the location of the effective scattering layer of turbulent medium along the line of sight on the base of analysis diffractive and refractive scintillation of pulsars. In some cases our estimations of distances to pulsars as more than 3 times differ from the values quoted in the TML pulsar catalog.
\end{abstract}

We will describe the turbulent medium by a characteristic scattering angle $\theta_{0}$ that corresponds to the scattering angle (radius) of a plane wave going out from the layer of turbulent medium. Below we consider 4 models of the distribution of the turbulent medium on the line of sight. Model 1: the medium occupies all space between a source and an observer. Model 2: the medium is located in the layer between a source and its boundary $r_{0}<<R$, where $\mathrm{R}$ - is the distance to pulsar. Model 3: the medium occupies the layer between an observer and the boundary $R-r_{0}<<R$. Model 4: the medium is concentrated in thin layer with thickness $\Delta r_{0}$ on the distance $r_{0}<R$ from the source, $\Delta r_{0}<<r_{0}, R$ (phase screen model). We concidered two types of spectra in turbulent medium: type 'a' spectrum is pure Kolmogorov one, the type ' $b$ ' is the piecewise power spectrum with a break at the inner scale 1. We supposed also that the temporal structure of scintillations is determined by the source moving with the velocity $V$. So we described the turbulent medium by next parameters: $\mathrm{R}, r_{0}, \theta_{0}$ and $\mathrm{V}$. For saturated scintillation we have 5 equations connecting these parameters with observables ones: $\theta_{\text {scat }}, T_{\text {ref }}, m_{\text {ref }}, t_{d i f}$, and $\Delta f_{d}$ (Smirnova, Shishov \&Stinebring 1998) but only 3 of them are independent. We can obtain using them

$$
R / \varphi=c G C^{2} /\left[\pi\left(T_{r e f} \Omega\right)^{2} \Delta f_{d}\right],
$$

where $\Omega$ is the visible angular velocity of the source which we used from TML catalog, $\mathrm{G}$ and $\mathrm{C}$ - coefficients depended on the medium model. The value $\varphi$ characterizes the medium distribution on the line of sight. For model 1 this value is $\varphi=1$, for models 2,3 and $4: \varphi=\left(r_{0} / R\right)^{2}\left(R / r_{e f f}\right)=r_{0} /\left(R-r_{0}\right)$. For model 2 it should be $\varphi<<1$ and for model 3: $\varphi>>1$. For model 2 and 3 only the value $R / \varphi=R^{2} / r_{0}$ or $R\left(R-r_{0}\right) / r_{0}$ can be obtained.

We determined the pulsar distances using the next algorithm. At first supposing the model 1 of the statistically homogeneous medium we calculated distance, $R_{0}$, using eq. (1) and then compared it with the distance, $R_{\text {cat }}$, from TML catalog of pulsars. These values are given in Table 1 . Values of $T_{\text {ref }}$ and $f_{d}$ we used from Stinebring et al. 1996. If angular velocity has a large error we gave the range of distances $R_{0}$. Estimation, $R_{\text {cat }}$, based on the TML model gives us the right evaluation of distance in statistical sense. But if we have some 
strong peculiarities in the electron density distribution in the line of sight to pulsar then we can obtain a big enough difference between $R_{0}$ and $R_{\text {cat }}$. When calculated distance, $R_{0}$, differed strongly (more than twice) from catalog values we conclude that we don't have the model 1 case. Then we excluded model 4 for these pulsars because the slope of structure function differs significantly from $\alpha=2$. For all of them we have the case of model 2 when $R_{0}>>R_{\text {cat }}$ or model 3 when $R_{0}<<R_{\text {cat }}$. For model 3 we count $\varphi \geq 2\left(r_{0} \geq 2 / 3 R\right)$ and so we have a lower limit on the distance $\mathrm{R}$. We used $R_{\min }$ value from HI absorption measurements as a distance for PSR $736-40$ and $1933+16$ and $R_{\text {cat }}$ value for PSR 1911-04. Calculated values of $\varphi$, accepted distance, $R$, calculated and measured modulation index, $m_{c a l}, m_{m}$ and type of model listed inTable 1 . For PSR 073640 we have $\varphi<<1$ and we know that Gum Nebula is in the line of sight to it on the distance about $0.5 \mathrm{kpc}$. We think that it is more reliable that PSR 0736-40 locates inside of this Nebula so we gave two possible variants of parameters in this case.

This work was supported by RFFI grant 97-02-17372 and INTAS-96-0154 project.

\section{References}

Taylor, J.H., Manchester R.N. \&Lyne, A.G. 1993, ApJ, v. 88, p.529

Stinebring, D.R. et al. 1996, Proc. in IAU Symp. 160, 455

Smirnova, T.V., Shishov, V.I. \&Stinebring, D.R. 1998, AZh, v. 42, p.766

Table 1. Used and defined parameters

\begin{tabular}{|c|c|c|c|c|c|c|c|c|}
\hline PSR & $\begin{array}{c}R_{\text {cat }}, \\
\mathrm{kpc}\end{array}$ & $\begin{array}{c}R_{0}, \\
\mathrm{kpc}\end{array}$ & Mod & $\begin{array}{c}1, \\
10^{10} \mathrm{~cm}\end{array}$ & $\varphi$ & $\begin{array}{c}\mathrm{R}, \\
\mathrm{kpc}\end{array}$ & $m_{\text {cal }}$ & $m_{m}$ \\
\hline $136+57$ & 2.89 & 2.52 & $1 \mathrm{a}$ & - & 1 & 2.5 & 0.15 & 0.15 \\
$329+54$ & 1.43 & 0.15 & $3 \mathrm{a}$ & - & $\geq 2$ & $\geq 0.9$ & 0.15 & 0.37 \\
$525+21$ & 2.3 & 2.85 & $1 \mathrm{a}$ & - & 0.9 & 2.9 & 0.27 & 0.31 \\
$531+21$ & 2 & 10.1 & $2 \mathrm{~b}$ & 30 & 0.058 & 2 & 0.32 & 0.32 \\
$736-40$ & $\geq 11$ & 19.1 & $2 \mathrm{a}$ & - & 0.017 & 2 & 0.024 & 0.03 \\
$736-40$ & $\geq 11$ & 19.1 & $2 \mathrm{a}$ & - & 0.004 & 0.5 & 0.026 & 0.03 \\
$740-28$ & 1.9 & 6.5 & $1 \mathrm{a}$ & - & 1 & 6.5 & 0.11 & 0.13 \\
$818-13$ & 2.46 & 0.2 & $3 \mathrm{a}$ & - & $\geq 2$ & $\geq 1.2$ & 0.13 & 0.23 \\
$833-45$ & 0.5 & 7.14 & $2 \mathrm{~b}$ & 60 & 0.015 & 0.5 & 0.23 & 0.24 \\
$1642-03$ & 2.9 & 2.1 & $1 \mathrm{a}$ & - & 1 & 2 & 0.31 & 0.46 \\
$1749-28$ & 1.5 & 1.1 & $1 \mathrm{a}$ & - & 1 & 1.1 & 0.22 & 0.25 \\
$1818-04$ & 2.1 & 1.6 & $1 \mathrm{a}$ & - & 1 & 1.6 & 0.1 & 0.12 \\
$1911-04$ & 3.2 & $16-122$ & $2 \mathrm{~b}$ & 60 & 0.057 & 3.2 & 0.19 & 0.22 \\
$1933+16$ & 7.9 & 3.5 & $2 \mathrm{~b}$ & 60 & 0.22 & 3.5 & 0.23 & 0.24 \\
$1946+35$ & 7.9 & $5.6-18$ & $1 \mathrm{a}$ & - & 1 & 5.6 & 0.057 & 0.055 \\
$2217+47$ & 2.45 & $.8-1.7$ & $1 \mathrm{a}$ & - & 1 & 1.7 & 0.26 & 0.3 \\
\hline
\end{tabular}

